\title{
Glucose repression of transcription of the Schizosaccharomyces pombe fbp1 gene occurs by a cAMP signaling pathway
}

\author{
Charles S. Hoffman ${ }^{1}$ and Fred Winston \\ Department of Genetics, Harvard Medical School, Boston, Massachusetts 02115 USA
}

\begin{abstract}
Transcription of the $f b p 1$ gene, encoding fructose-1,6-bisphosphatase, of Schizosaccharomyces pombe is subject to glucose repression. Previous work has demonstrated that several genes (git genes) are required for this repression. In this report we demonstrate that one of these genes, git2, is the same as the cyr1 gene, which encodes adenylate cyclase, and that loss-of-function mutations in git2 cause constitutive $f b p 1$ transcription. Addition of cAMP to the growth medium suppresses the transcriptional defect in git2 mutants as well as in strains that carry mutations in any of six additional git genes. Similarly, exogenous cAMP represses $f b p 1$ transcription in wild-type cells grown on a derepressing carbon source. Different levels of adenylate cyclase activity in different git 2 mutants, coupled with the result that some git 2 mutants display intragenic complementation, strongly suggest that adenylate cyclase acts as a multimer and that different git2 mutations alter distinct activities of adenylate cyclase, including catalytic activity and response to glucose. Additional experiments demonstrate that this cAMP signaling pathway is independent of the $S$. pombe ras1 gene and works by activation of cAMP-dependent protein kinase.
\end{abstract}

[Key Words: Schizosaccharomyces pombe; fructose-bisphosphatase; glucose repression; cAMP; adenylate cyclase; transcription]

Received January 4, 1991; accepted January 15, 1991.

Cells regulate transcription in response to signals from their environment. For example, unicellular organisms transcribe specific genes to metabolize the carbon sources available. If glucose is also present, however, many genes required for the utilization of other carbon sources are no longer expressed. This phenomenon has been termed glucose repression or catabolite repression (Magasanik 1961).

Glucose repression appears to work through different mechanisms in the bacterium Escherichia coli and the yeast Saccharomyces cerevisiae. In E. coli, glucose causes a reduction in the level of cAMP that binds to and is required for activity of the catabolite gene activator protein (for review, see Botsford 1981). In S. cerevisiae, however, cAMP is not required for expression of genes subject to glucose repression (Matsumoto et al. 1982b, 1983) and cAMP levels are not reduced under conditions that derepress expression of such genes (Eraso and Gancedo 1984). In contrast, S. cerevisiae ADH2 transcription appears to be repressed by cAMP through the phosphorylation and inactivation of the transcriptional activator ADR1 by cAMP-dependent protein kinase (cAPK; Cherry et al. 1989; Taylor and Young 1990). In S. cerevisiae, glucose repression involves a variety of mechanisms, as different mutations have been isolated that

${ }^{1}$ Present address: Department of Biology, Boston College, Chestnut Hill, Massachusetts 02167 USA. allow constitutive expression of distinct subsets of glucose repressible genes (for review, see Gancedo and Gancedo 1986; Johnston 1987).

In the yeast Schizosaccharomyces pombe, the $f b p 1$ gene, encoding the gluconeogenic enzyme fructose-1,6bisphosphatase, is subject to glucose repression (Vassarotti and Friesen 1985; Hoffman and Winston 1989, 1990). We have observed that the level of $f b p 1$ transcription can vary over a range of $>200$-fold with respect to the carbon source. Using a pair of translational fusions to $f b p 1$, we have isolated mutants in which transcription of an $f b p 1-u r a 4$ fusion is constitutive. These mutations identified 10 genes, designated git (glucose-insensitive transcription) (Hoffman and Winston 1990). Mutations in 8 of these 10 genes cause a significant increase in $f b p 1$ transcription under normally repressing conditions (see Fig. 2, below; Hoffman and Winston 1990). We have begun a molecular analysis of glucose repression by cloning and analyzing one of these git genes.

In this report we demonstrate that the git2 gene, mutations in which allow high-level constitutive $f b p 1$ transcription, is identical to the $c y r 1$ gene, which encodes adenylate cyclase (Yamawaki-Kataoka et al. 1989; Young et al. 1989|. This enzyme functions to convert ATP to cAMP in response to external stimuli and is involved in many signaling pathways in eukaryotic cells (Gilman 1984; Levitzki 1988). In these signaling pathways the role of CAMP is to activate CAPK, which is composed of 
a pair of regulatory subunits associated with a pair of catalytic subunits. cAMP binds the regulatory subunits of cAPK, causing them to dissociate from and thereby activate the catalytic subunits of CAPK, which are now able to phosphorylate target proteins (Beebe and Corbin 1986; Edelman et al. 1987). Some known target proteins are enzymes that are inactivated by phosphorylation, whereas others are transcriptional activators (Beebe and Corbin 1986; Roesler et al. 1988).

In S. cerevisiae, many genes have been identified that are involved in the adenylate cyclase pathway. Adenylate cyclase is encoded by the CYR1 gene (Casperson et al. 1985), whereas the upstream activators are encoded by RAS1 and RAS2 (Broek et al. 1985; Toda et al. 1985). $C D C 25$ encodes a positive regulator of RAS activity required for adenylate cyclase activation (Broek et al. 1987; Robinson et al. 1987), whereas IRA1 (Tanaka et al. 1989) and IRA2 (Tanaka et al. 1990) encode negative regulators of RAS activity. The CYR1 gene product is associated with a $70-\mathrm{kD}$ protein encoded by the CAP/SRV2 gene (Fedor-Chaiken et al. 1990; Field et al. 1990). The 70-kD protein is also required for RAS activation of adenylate cyclase. The regulatory subunit of CAPK is encoded by $B C Y 1$, mutations in which bypass the requirement for active adenylate cyclase (Matsumoto et al. 1982a). The catalytic subunits of cAPK are encoded by each of the TPK1, TPK2, and TPK3 genes (Toda et al. 1987b).

In $S$. pombe, less is known about the adenylate cyclase pathway. The genes that encode adenylate cyclase (cyr1; Yamawaki-Kataoka et al. 1989; Young et al. 1989) and the regulatory subunit of cAPK (cgs1; M. McLeod, pers. comm.) have been identified. An S. pombe RAS gene homolog, ras1, has been identified (Fukui and Kaziro 1985; Nadin-Davis et al. 1986a); however, in contrast to $S$. cerevisiae, ras 1 does not appear to stimulate adenylate cyclase activity in $S$. pombe (Fukui et al. 1986; NadinDavis et al. 1986b; for review, see Nadin-Davis et al. 1989).

Additional experiments in this report demonstrate that git2 (cyr1), other git genes, and cgs1 are required for normal glucose-mediated regulation of $f b p 1$ transcription. As expected, ras1 does not play a role in $f b p 1$ regulation. In addition, intragenic complementation between various git2 mutant alleles and differential effects on adenylate cyclase activity (as assayed in vitro) and cAMP levels by these mutations indicate that adenylate cyclase is a multimer and that git2 mutations cause constitutive $f b p 1$ transcription by altering distinct functions of the protein. These results lead to the conclusion that $f b p 1$ transcription is regulated by a cAMP signaling pathway, independent of ras1, and that the role of the cAMP signal in $S$. pombe glucose repression is to activate cAPK.

\section{Results}

Cloning of the git2 gene as a high copy suppressor of a gitl mutation

Previous work had identified a large number of git genes required for glucose repression of $f b p 1$ transcription (Hoffman and Winston 1990). To initiate a molecular analysis of the git genes, we set out to clone the git1 gene. Mutations in git 1 allow constitutive transcription of the wild-type $f b p 1$ gene, as well as constitutive expression of both $f b p 1-u r a 4$ and $f b p 1-1 a c Z$ translational fusions, under normally repressing conditions (Hoffman and Winston 1990). Because cells that express the $f b p 1-u r a 4$ fusion are sensitive to the pyrimidine analog 5-fluoro-orotic acid (5FOA; Boeke et al. 1984; Hoffman and Winston 1990), git1- mutants that contain the fbp1-ura4 fusion are 5FOA sensitive $\left(5 \mathrm{FOA}^{\mathrm{s}}\right.$; $\mathrm{Git}^{-}{ }^{-}$phenotype) when grown under repressing conditions $(8 \%$ glucose as the carbon source); git $1^{+}$strains are $5 \mathrm{FOA}$ resistant $\left(5 \mathrm{FOA}^{\mathrm{R}} ; \mathrm{Git}^{+}\right.$phenotype $)$. We therefore screened an $S$. pombe genomic library in a high copy number vector for plasmids that could transform a git $1^{-}$ fbp1-ura4 strain (Git ${ }^{-}$) to $\mathrm{Git}^{+}$(see Materials and methods). After screening 17,000 transformants, we obtained one plasmid (pCHY26) that conferred a strong $\mathrm{Git}^{+}$ $\left(5 \mathrm{FOA}^{\mathrm{R}}\right)$ phenotype.

Three pieces of evidence demonstrated that pCHY26 encodes git2 rather than git1. First, when pCHY26 was integrated into a git ${ }^{-}$strain, the integrant unexpectedly remained $\mathrm{Git}^{-}$, demonstrating that the cloned DNA, when present in low copy number, could not complement the git1-1 mutation. Second, by tetrad analysis, the LEU2 gene on pCHY26 was unlinked to git1, demonstrating that the plasmid had not integrated at the git1 locus. Third, additional crosses by other git mutants demonstrated that $\mathrm{pCHY} 26$ had directed integration to the git2 locus (see Materials and methods). To rule out the possibility that plasmid pCHY26 had integrated by nonhomologous recombination at a site fortuitously linked to git2, we also integrated a smaller derivative of pCHY26, pCHY27 (Fig. 1), and demonstrated by Southern hybridization analysis and linkage analysis that it had integrated by homologous recombination at the git2 locus (data not shown). Therefore, pCHY26 carries the git2 gene, and git2 is a high copy number suppressor of a git1 mutation.

Because git2 is a high copy number suppressor of a git1 mutation, we transformed strains carrying mutations in git1-git10 (FWP113, CHP210, CHP200, CHP17, CHP75, CHP261, CHP235, CHP60, CHP232, CHP201) with pCHY26 to determine whether other git mutations could be suppressed by git 2 in high copy number. Because each strain also contained the $f b p 1-u r a 4$ fusion, suppression was assayed by monitoring the 5FOA phenotype of each transformant. Strains that carry a mutation in git1, git2, git3, git5, git7, git8, or git10 were suppressed by high copy number git2 $\left(5 \mathrm{FOA}^{\mathrm{R}}\right)$, whereas transformants carrying a mutation in git4, git6, or git 9 were not suppressed $\left(5 \mathrm{FOA}^{\mathrm{s}}\right)$. These results suggest that the git2 gene product may act downstream of the gene products of git1, git3, git5, git7, git8, and git10. The gene products of git4, git6, and git9 may act either downstream of or in a separate pathway from the git2 gene product.

\section{The git 2 gene encodes adenylate cyclase}

Molecular analysis of the cloned git2 gene has shown 


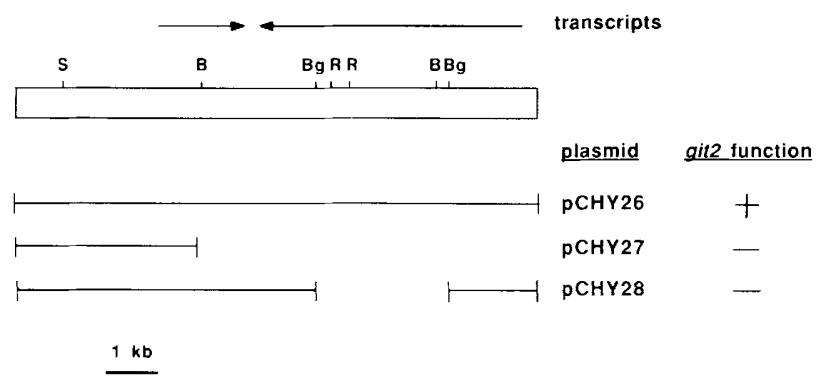

Figure 1. Restriction map, transcription units, and subclones of the git2 clone. The insert DNA present in the original git2 clone, pCHY26, and two nonfunctional subclones are shown. The insert DNA in pCHY26 is an $\sim 10.5-\mathrm{kb}$ HindIII fragment, with additional internal HindIII sites not shown. SalI (S), $B a m H I(B), B g l I I(B g)$, and EcoRI (R) sites are marked. Function was determined by the ability of the plasmid to transform a git2 mutant strain from $5 \mathrm{FOA}^{\mathrm{S}}$ to $5 \mathrm{FOA}^{\mathrm{R}}$. The size, orientation, and approximate positions of the two transcripts that hybridize to pCHY 26 were determined by a series of Northern hybridization analyses (data not shown). The longer transcript (right) is the git2 transcript.

that it is identical to the previously identified cyr1 gene, which encodes adenylate cyclase (Yamawaki-Kataoka et al. 1989; Young et al. 1989). Plasmid pCHY28 (Fig. 1), a derivative of pCHY26 in which a $2.8-\mathrm{kb}$ BglII fragment has been removed, was unable to transform git2 mutant strains to $\mathrm{Git}^{+}$. We, therefore, assumed that the git2 gene must overlap some, if not all, of this portion of the clone and initiated DNA sequencing around the leftmost BglII site (Fig. 1).

Determination of $340 \mathrm{bp}$ of DNA sequence gave a sequence identical to that of part of the coding region for the adenylate cyclase catalytic domain obtained by Yamawaki-Kataoka et al. (1989; EMBL/GenBank file name Yspcyrla.pl; base pairs 4084-4235 and 4261-4448) and by Young et al. (1989; EMBL/GenBank file name Yspadc.pl; base pairs 5485-5636 and 5662-5849|. Restriction mapping of pCHY26 with nine restriction endonucleases (data not shown) was consistent with our conclusion that git2 and cyr1 are the same gene.

To verify that git2 and cyr1 are the same gene and to determine whether the $\mathrm{Git}^{-}$phenotype can be caused by loss of adenylate cyclase activity, we constructed git2 (cyr1) null strains. In these $S$. pombe strains, FWP190 and FWP191, the wild-type gene is replaced by DNA that contains a deletion of a BgIII fragment internal to git2 (see Materials and methods; Fig. 1). This deletion mutation, designated git2-1, removes part of the coding region for the catalytic domain of adenylate cyclase and should cause the remainder of the gene to be translated out of frame. Northern hybridization analysis of RNA from strain FWP191 indicates that git2-1 only alters the 5400nucleotide git2 (cyr1) transcript and has no effect on a 1600-nucleotide transcript $>700 \mathrm{bp}$ from the BglII deletion (data not shown). Therefore, the git2 (cyr1) gene is the only gene affected by the BglII deletion.
Strains that contain the git2-1 null allele display a $\mathrm{Git}^{-}$phenotype and constitutively express the fbp1lacZ fusion (FWP191; Table 1). Membrane extracts made from strain FWP191 (git2-1) lack any detectable adenylate cyclase activity (Table 2). Therefore, loss of git2 function (adenylate cyclase) causes a $\mathrm{Git}^{-}$phenotype. This null allele fails to complement other git2 mutant alleles, confirming that the other git 2 mutations are in the cyr 1 gene. In contrast to $S$. cerevisiae, $S$. pombe haploid strains that contain a null mutation in git2 (cyr1) are viable in the absence of cAMP. This result has also been observed by Maeda et al. (1990) and Kawamukai et al. (1991).

cAMP represses fbp 1 transcription in git mutant and wild-type strains

Because adenylate cyclase acts to convert ATP to cAMP, and as mutations in the gene that encodes adenylate cyclase cause constitutive transcription of the $f b p 1$ gene, we examined the effect of exogenous cAMP on transcription of the $f b p 1$ gene. Our results demonstrate that in most git mutants and in wild-type strains, cAMP alters $f b p 1$ transcription. The addition of $5 \mathrm{~mm}$ cAMP represses transcription of the $f b p 1$ gene in a git2 mutant strain as well as in git1, git3, git5, git7, git8, or git10 mutants (Fig. 2). Exogenous cAMP, however, has no detectable effect on $f b p 1$ transcription in a git6 mutant (Fig. 2). Because the git4 and git 9 mutations do not cause a detectable increase in $f b p 1$ transcription, we were unable to determine whether there is an effect of addition of exogenous cAMP on $f b p 1$ transcription in git 4 or git 9 mutants.

Expression of an $f b p 1-l a c Z$ fusion in most git mutants is also repressed by cAMP (Table 1). $\beta$-Galactosidase assay results demonstrate that the addition of cAMP sup-

Table 1. fbpl-lacZ expression in git mutants \pm cAMP

\begin{tabular}{llcc}
\hline & & \multicolumn{2}{c}{$\begin{array}{c}\text { B-Galactosidase activity } \\
\text { in } 8 \% \text { glucose }^{\mathbf{a}}\end{array}$} \\
\cline { 3 - 4 } Strain & $\begin{array}{l}\text { git } \\
\text { genotype }\end{array}$ & -cAMP & + cAMP \\
\hline FWP70 & git & $17 \pm 5$ & $13 \pm 3$ \\
FWP134 & git1-1 & $1590 \pm 121$ & $34 \pm 3$ \\
FWP191 & git2-1 & $3486 \pm 432$ & $24 \pm 4$ \\
FWP135 & git2-210 & $1994 \pm 354$ & $27 \pm 5$ \\
FWP136 & git3-200 & $505 \pm 107$ & $37 \pm 15$ \\
FWP173 & git4-17 & $39 \pm 11$ & $36 \pm 3$ \\
FWP174 & git5-75 & $848 \pm 161$ & $21 \pm 3$ \\
FWP139 & git6-261 & $2396 \pm 71$ & $1674 \pm 114$ \\
FWP140 & git7-235 & $1672 \pm 301$ & $94 \pm 11$ \\
FWP175 & git8-60 & $859 \pm 195$ & $20 \pm 3$ \\
FWP142 & git9-232 & $100 \pm 27$ & $103 \pm 12$ \\
FWP143 & git10-201 & $1106 \pm 321$ & $20 \pm 2$ \\
\hline
\end{tabular}

The values given represent $\beta$-galactosidase sp. act. \pm S.E. for two to four independent cultures. Strains were grown overnight to $1 \times 10^{7}$ cells $/ \mathrm{ml}$ in YEL ( $8 \%$ glucose) in the presence or absence of $5 \mathrm{mM}$ cAMP and assayed. 
Table 2. Adenylate cyclase activity and cAMP levels in git2 mutants

\begin{tabular}{|c|c|c|c|c|}
\hline \multirow[b]{2}{*}{ Strain } & \multirow{2}{*}{$\begin{array}{l}\text { git2 } \\
\text { allele }\end{array}$} & \multirow{2}{*}{$\begin{array}{l}\text { Adenylate } \\
\text { cyclase } \\
\text { activity }^{\mathrm{a}}\end{array}$} & \multicolumn{2}{|c|}{ cAMP levels $^{\mathrm{b}}$} \\
\hline & & & repressed & derepressed \\
\hline FWP70 & git2 $^{+}$ & $10.4 \pm 0.3$ & $3.6 \pm 0.1$ & $3.4 \pm 0.6$ \\
\hline FWP135 & git2-210 & $8.5 \pm 1.5$ & $2.5 \pm 0.3$ & ND \\
\hline FWP182 & git2-7 & $8.6 \pm 0.8$ & $5.7 \pm 0.1$ & ND \\
\hline FWP183 & git2-13 & $0.3 \pm 0.2$ & $0.9 \pm 0.1$ & ND \\
\hline FWP184 & git2-61 & $1.4 \pm 0.5$ & $2.8 \pm 0.2$ & ND \\
\hline FWP191 & git2-1 & $<0.1$ & $0.4 \pm 0.1$ & ND \\
\hline
\end{tabular}

The values given represent sp, act. \pm S.E. for three to four independent cultures assayed as described in Materials and methods. One unit of adenylate cyclase activity represents the production of $1 \mathrm{pmole}$ of $\mathrm{cAMP} / \mathrm{min}$ per $\mathrm{mg}$ of protein.

${ }^{b}$ cAMP levels were measured as described in Materials and methods for cultures grown in PM medium under repressing ( $8 \%$ glucose $)$ or derepressing $(0.1 \%$ glucose $+3 \%$ glycerol $)$ conditions. Values given represent pmoles of $\mathrm{cAMP} / \mathrm{mg}$ of total protein \pm S.E. for two independent cultures. The low value for cAMP measurements in the git2-1 strain was still observed after treatment of the extract with phosphodiesterase (data not shown). Therefore, this strain does not produce any detectable cAMP. (ND) Not determined.

presses the constitutive expression of the $f b p 1-l a c Z$ fusion in the same set of git mutants as indicated by the Northern blot analysis of the $f b p 1$ transcript. Exogenous cAMP has little or no effect on $\beta$-galactosidase activity in git4, git6, or git9 mutant strains.

Addition of exogenous cAMP also represses $f b p 1$ transcription in both git ${ }^{+}$and git2-210 strains grown under derepressing conditions (Fig. 3). Similarly, cAMP addition represses expression of an $f b p 1-l a c Z$ fusion in a git $^{+}$ strain (FWP70) grown under derepressing conditions: This strain contained $1549 \pm 47$ units of $\beta$-galactosidase activity (sp. act. \pm S.E. for four independent liquid cultures) in the absence of cAMP and only $122 \pm 25$ units in the presence of cAMP. In addition, cAMP caused cell death when FWP70 was grown in the presence of $0.1 \%$ glucose and 3\% glycerol (data not shown), probably due to the inability of the cells to use glycerol as a carbon source owing to the simulation of glucose repression by cAMP. These results demonstrate that exogenous cAMP is sufficient for repression of transcription from the $f b p 1$ promoter in a wild-type strain grown under normally derepressing conditions.

\section{git2 intragenic complementation}

We determined previously that diploids formed from strains carrying the git2-7 and git2-61 alleles have a Git ${ }^{+}$ phenotype, indicating intragenic complementation (Hoffman and Winston 1990). Therefore, strains that contain the git2-7 and git2-61 alleles have been tested for their ability to complement strains carrying 31 independent git2 mutations. Diploids carrying either the git2-7 or git2-61 allele on one homolog and each of the 31 git2 alleles on the other homolog were tested for complemen- tation as described in Materials and methods. We also tested all combinations of the 17 original $h^{-}$git 2 isolates by the 14 original $h^{+}$git2 isolates for intragenic complementation.

On the basis of these complementation tests, 11 of the 31 git 2 mutants display at least some intragenic complementation. Eight members form one group, and three members form the second group. The remaining 20 spontaneous git2 mutant alleles, as well as the git2-1 null allele, do not complement any git2 allele. The first complementation group consists of three strongly complementing alleles, git2-7, git2-110, and git2-266, four moderately complementing alleles, git2-18, git2-68, git2-216, and git2-226, and one weakly complementing allele, git2-210. The second complementation group consists of the strongly complementing allele git2-61 and two moderately complementing alleles, git2-29 and git2-83. An example of the complementation analysis is shown in Figure 4. Because most spontaneous git2 mutant alleles do not complement alleles of either complementation group, these groups represent intragenically complementing alleles and not mutations in two tightly linked genes.

\section{Adenylate cyclase activity and cAMP levels in git2 mutant strains}

To determine whether all git2 mutations cause a loss or reduction of adenylate cyclase activity, adenylate cyclase activity was assayed in membrane extracts from git $^{+}$and a set of git2 mutant strains. These assays were performed in the presence of $\mathrm{Mn}^{2+}$, as in vitro activation of $S$. pombe adenylate cyclase in the presence of $\mathrm{Mg}^{2+}$ has not been observed (Yamawaki-Kataoka et al. 1989; Engelberg et al. 1990; C.S. Hoffman and F. Winston, unpubl.). The results of the assays (Table 2) show that the git2-61 and git2-13 mutations significantly reduce adenylate cyclase activity, whereas the git2-1 null allele causes the complete loss of adenylate cyclase activity. The git2-7 and git2-210 mutations, however, have little or no effect on adenylate cyclase activity as measured in vitro. Although the four spontaneous git2 mutations have different effects on adenylate cyclase activity, they all increase $f b p 1-1 a c Z$ expression to a similar high constitutive level (data not shown). Therefore, git2 alleles that confer very different levels of adenylate cyclase activity as measured in vitro all confer a similar $\mathrm{Git}^{-}$phenotype in vivo.

To further analyze the different classes of git2 mutants, we also measured steady-state cAMP levels in wild-type and git2 mutant strains (Table 2). The git2-1 (null) and git2-13 mutants both have greatly reduced levels of cAMP. (The low value that we obtained for cAMP measurements in the git2-1 strain was still observed even after treatment of the extract with phosphodiesterase (data not shown). Therefore, this strain does not produce any detectable cAMP.) The other git2 mutants tested do not have significantly reduced cAMP levels. In addition, steady-state cAMP levels in a git2 ${ }^{+}$strain are similar for growth under conditions repressing or dere- 


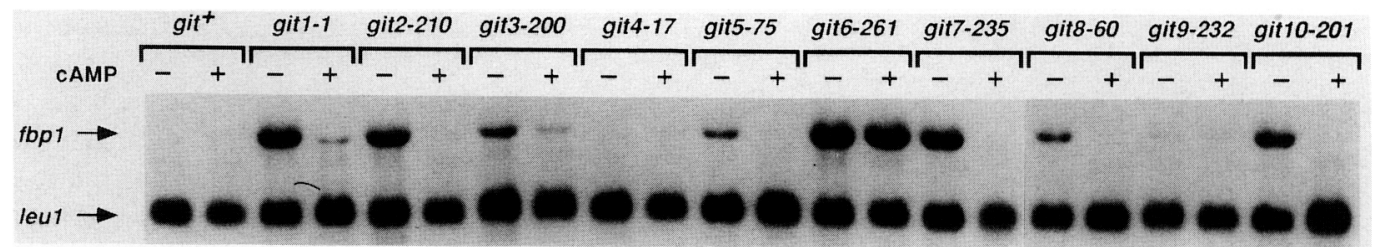

Figure 2. Exogenous cAMP represses $f b p 1$ transcription in most git mutant strains. Northern hybridization analysis was performed on RNA from a git ${ }^{+}$strain (FWP70) and from strains carrying mutations in git1-git10 (FWP134, FWP135, FWP136, FWP173, FWP174, FWP139, FWP140, FWP175, FWP142, and FWP143; see Table 1 for corresponding fbp1-lacZ expression), grown under repressing conditions ( $8 \%$ glucose) in the presence and absence of $5 \mathrm{mM}$ cAMP. The filter was hybridized to ${ }^{32} \mathrm{P}$-labeled plasmids pAV06 (to detect fbp1 RNA; Vassarotti and Friesen 1985) and pYK311 (to detect leu1 RNA as an internal standard; Kikuchi et al. 1988), and exposed to X-ray film.

pressing for $f b p 1$ expression. Therefore, although some git2 alleles abolish or reduce cAMP levels and cause a Git $^{-}$phenotype, some other git2 alleles cause a Git $^{-}$ phenotype, although they cause little change in steadystate cAMP levels.

\section{fbpl transcription is controlled by a cAMP-dependent protein kinase}

In other eukaryotic cAMP signaling pathways, the role of cAMP is to activate cAMP-dependent protein kinase (cAPK), which then phosphorylates target proteins (Beebe and Corbin 1986). To determine whether the role of cAMP in $S$. pombe glucose repression is to activate cAPK, we examined $f b p 1$ transcription in a strain containing a mutation in the cgs1 gene that encodes the regulatory subunit of cAPK (M. McLeod, pers. comm.). On the basis of constitutive transcription of $f b p 1$ in a strain lacking adenylate cyclase activity, if $\operatorname{cgs} 1$ is in the same pathway, a cgs1 mutation would be expected to reduce or eliminate $f b p 1$ expression in derepressed cells. For this experiment we used a mutant that contains the spontaneous cgs1-1 mutation (M. McLeod, pers. comm.). The results demonstrate that $c g s 1-1$ reduces $f b p 1$ transcription in a strain grown under derepressing conditions (0.1\% glucose and $3 \%$ glycerol; Fig. 5). Furthermore, the cgs1-1 mutation greatly reduces $f b p 1-l a c Z$ expression in

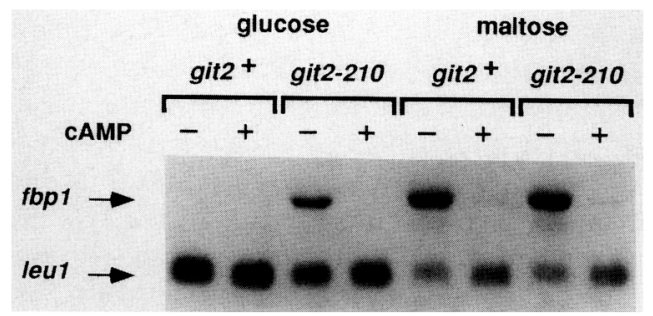

Figure 3. Exogenous cAMP represses $f b p 1$ transcription in a git2 $^{+}$and a git $2^{-}$mutant strain grown on a derepressing carbon source. Northern hybridization analysis was performed on RNA from FWP70 (git2 ${ }^{+}$) and from FWP135 (git2-210) grown on a repressing carbon source ( $8 \%$ glucose) and a derepressing carbon source $(3 \%$ maltose), in the presence and absence of $5 \mathrm{~mm}$ cAMP, and probed as described in Fig. 2. strains grown under derepressing conditions $13 \% \mathrm{mal}-$ tose; Table 3). These results strongly suggest that cAMP represses $f b p 1$ transcription by the activation of cAPK. The more dramatic effect of the cgs1 mutation observed in Table 3, relative to Figure 5, could be due to the fact that $3 \%$ maltose is a less derepressing carbon source than $0.1 \%$ glucose plus $3 \%$ glycerol.

To characterize the role of $\operatorname{cgs} 1$ in this pathway in greater detail, we carried out epistasis tests with cgs1 and git2, and with cgs1 and git6. As expected, the cgs1-1 git2-1 double mutant resembled the cgs1-1 mutant (Table 3 ), that is, the cgs1-1 mutation suppressed the constitutive expression of the $f b p 1-1 a c Z$ fusion conferred by the git2-1 allele. This result supports the idea that glucose repression of $f b p 1$ is mediated by cAPK. Interestingly, the cgs1-1 git6-261 double mutant is constitutive for expression of $f b p 1-l a c Z$, consistent with the observation that the git6-261 mutation is not suppressed by exogenous CAMP. This epistasis test suggests that the

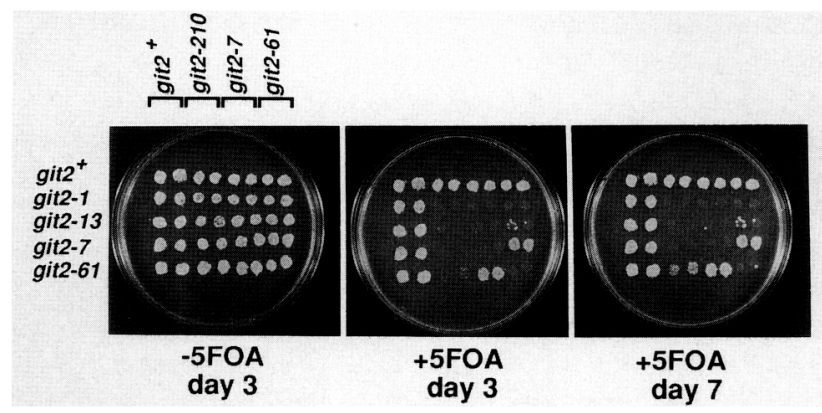

Figure 4. Certain git2 mutant alleles display strong intragenic complementation. Complementation analyses were performed as described in Materials and methods. Two diploids from each cross were colony-purified and replica-plated to media with and without $5 \mathrm{FOA}$ at $30^{\circ} \mathrm{C}$. Diploids that contain a git2 ${ }^{+}$allele are $5 \mathrm{FOA}^{\mathrm{R}}$, demonstrating that the git2 mutations are recessive. Diploids that contain two git2 mutations are usually 5 FOA ${ }^{\mathrm{s}}$ (noncomplementation), except those that contain git2-7 with git2-61, which are 5 FOA $^{\mathrm{R}}$ (growth on 5 FOA by day 3 ; strong complementation) and those that contain git2-210 with git2-61, which are weakly 5 FOA $^{\mathrm{R}}$ (growth on 5 FOA by day 7 ; weak complementation). Rare, individual $5 \mathrm{FOA}^{\mathrm{R}}$ colonies that appear in $5 \mathrm{FOA}^{\mathrm{S}}$ patches likely represent events other than complementation, such as mitotic recombination at git2, second site suppressor mutations, or reversion events. 


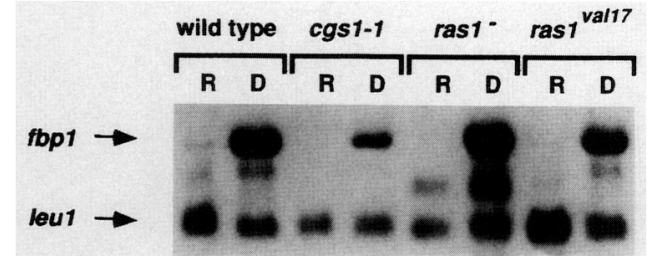

Figure 5. $f b p 1$ transcription is reduced in a $c g s 1$ mutant strain and is normal in strains carrying ras1 mutations. Northern hybridization analysis was performed on RNA from strains FWP180 (cgs1 ${ }^{+}$ras1 $^{+}$), FWP177 (cgs1-1), FWP179 (ras1 ${ }^{-}$), and FWP181 (ras1 $1^{\text {val17 }}$; activated form) grown under repressing (8\% glucose) and derepressing $\{0.1 \%$ glucose and $3 \%$ glycerol $\}$ conditions. Additional bands present in lanes containing FWP180, FWP179, and FWP181 RNAs are probably due to transcription of the $L E U 2$ gene integrated adjacent to ras1.

git6 gene product does not normally participate in the stimulation of adenylate cyclase but, rather, acts more directly in repression of $f b p 1$ transcription.

\section{Mutations in rasl do not alter fbpl transcription}

$S$. cerevisiae adenylate cyclase is activated by the products of the RAS1 and RAS2 genes (Toda et al. 1985). In $S$. pombe, however, experiments indicate that the ras 1 gene does not carry out a similar function (Fukui et al. 1986; Nadin-Davis et al. 1986b, 1989). To investigate further the role of ras1 with respect to adenylate cyclase activity, we examined $f b p 1$ transcription in strains that contain either a ras $1^{-}$or a ras $1^{\text {val17 }}$ (activated) mutation (Nadin-Davis et al. 1986b). Northern hybridization analysis (Fig. 5) demonstrates that there is wild-type regulation of $f b p 1$ transcription in both strains. This result demonstrates further that $S$. pombe ras 1 does not regulate adenylate cyclase activity.

\section{Discussion}

Our previous work identified a set of genes required for glucose repression of $f b p 1$ transcription in $S$. pombe

Table 3. fbp1-lacZ expression in cgs1-1, cgs1-1 git2-1 and cgs1-1 git6-261 mutants

\begin{tabular}{llcc}
\hline & \multirow{2}{*}{$\begin{array}{l}\text { Relevant } \\
\text { Strain }\end{array}$} & \multicolumn{2}{c}{$\beta$-Galactosidase activity $^{\mathrm{a}}$} \\
\cline { 3 - 4 } & genotype & $\mathbf{8 \%}$ glucose & $\mathbf{3} \%$ maltose \\
\hline FWP189 & cgs1 $^{+}$git $^{+}$ & $12 \pm 0$ & $1508 \pm 212$ \\
FWP191 & git2-1 $^{\text {Fit6-261 }}$ & $3486 \pm 432$ & $5444 \pm 1043$ \\
FWP139 & git-1 & $2396 \pm 71$ & $7400 \pm 2413$ \\
FWP192 & cgs1-1 & $21 \pm 6$ & $118 \pm 8$ \\
FWP193 & cgs1-1 git2-1 & $18 \pm 5$ & $116 \pm 14$ \\
FWP194 & cgs1-1 git6-261 & $3417 \pm 264$ & $4621 \pm 1078$ \\
\hline
\end{tabular}

${ }^{a}$ The values given represent $\beta$-galactosidase sp. act. \pm S.E. for two or three independent cultures. Strains were grown overnight in YEL to $1 \times 10^{7}$ cells $/ \mathrm{ml}$, with either $8 \%$ glucose $/$ repressing) or $3 \%$ maltose (derepressing) as the carbon source, and assayed.
(Hoffman and Winston 1990). Our present work demonstrates that one of these genes, git2, is the same as the previously identified cyr1 gene, encoding adenylate cyclase, and that cells that lack this activity are unable to repress $f b p 1$ transcription. Our experiments also demonstrate that exogenous cAMP represses $f b p 1$ transcription in both wild-type and many, but not all, git mutant strains. These results strongly suggest that $S$. pombe responds to glucose by activating adenylate cyclase as part of a cAMP signaling pathway.

The adenylate cyclase in vitro assays performed here almost certainly represent the unstimulated activity of adenylate cyclase and do not reflect the ability of adenylate cyclase to respond to in vivo stimuli. Adenylate cyclase activity from $S$. pombe is not stimulated by the addition of guanine nucleotides in vitro, and activity has only been observed when assayed in the presence of $\mathrm{Mn}^{2+}$; activity in the presence of $\mathrm{Mg}^{2+}$ has not been detected (Yamawaki-Kataoka et al. 1989; Engelberg et al. 1990; C.S. Hoffman and F. Winston, unpubl.). Our results suggest that the addition of glucose may cause a post-translational modification of adenylate cyclase that stimulates activity in vivo but that is not detectable by current in vitro assays. Clearly, an in vitro assay that reflects more clearly in vivo activity and stimulation will be necessary to allow a more accurate assessment of the adenylate cyclase defects in the different git2 mutants.

Differences in the adenylate cyclase levels among git2 mutants and the observation of git2 intragenic complementation suggest that $S$. pombe adenylate cyclase contains separable domains and that it functions in a complex containing two or more copies of the git2 (cyr1) gene product. Genetic evidence for such a complex in $S$. cerevisiae has been obtained by Field et al. (1990b). That mutations in each git2 intragenic complementation group represent alterations of different functional domains of adenylate cyclase is consistent with results of adenylate cyclase assays for different git2 mutants (Table 2 ). Two strains that contain mutations in the first complementation group (git2-7 and git2-210) possess virtually wild-type levels of adenylate cyclase activity as measured in vitro and are therefore unlikely to affect directly the adenylate cyclase catalytic domain. In contrast, a strain that contains a mutation in the second complementation group (git2-61) possesses significantly reduced adenylate cyclase activity as does a strain that contains a mutation that fails to complement members of either group (git2-13).

The cAMP levels in some git2 mutants are reduced relative to that of a wild-type strain (Table 2), supporting the hypothesis that these mutations cause a reduction in the ability of the cell to produce cAMP in response to glucose. The facts that a git 2 null mutant, which makes no detectable cAMP, causes derepression of $f b p 1$ transcription, and that addition of exogenous cAMP restores repression, demonstrate that cAMP causes $f b p 1$ repression. The result that some git2 mutants do not have reduced cAMP levels may reflect feedback control of cAMP levels as in S. cerevisiae (Nikawa et al. 1987). In 
addition, wild-type cultures grown under repressing and derepressing conditions possess similar steady-state levels of cAMP. These results suggest that the steady-state level of cAMP is not altered by glucose repression. Rather, the cAMP signal is likely to be transient in cells exposed to glucose, as is the case in S. cerevisiae (Mbonyi et al. 1988; Thevelein 1988). Measurement of cAMP levels immediately after glucose addition in wild-type and git strains will test whether cAMP levels are altered in response to glucose.

These studies provide us with likely candidates for genes required for the activation of adenylate cyclase in S. pombe. These candidates include the genes git1, git3, git5, git7, git8, and git10, as the $\mathrm{Git}^{-}$phenotype caused by mutations in these genes is suppressed by exogenous cAMP and by the git2 gene in high copy number. One or more of these git genes may encode subunits of a trimeric guanine nucleotide-binding protein (G protein) that stimulates adenylate cyclase activity, as has been observed for mammalian cells (Gilman 1984). Because the $\mathrm{G}$ protein may have to assemble and/or couple to the glucose receptor before stimulation of adenylate cyclase, mutations in any of the genes encoding $G$ protein subunits could produce a Git ${ }^{-}$phenotype. One or more git genes may also encode a glucose receptor, proteins that modulate G protein activity [analogous to $C D C 25$ of $S$. cerevisiae (Broek et al. 1987; Robinson et al. 1987)], or additional subunits of the active adenylate cyclase complex [analogous to the 70-kD adenylate cyclase-associated protein of $S$. cerevisiae (Fedor-Chaiken et al. 1990; Field et al. 1990a)]. Alternatively, stimulation of adenylate cyclase in $S$. pombe may occur by a different type of pathway.

Glucose repression of $f b p 1$ in $S$. pombe may be analogous in some ways to glucose repression of $A D H 2$ in $S$. cerevisiae. In $S$. cerevisiae, studies have demonstrated that glucose induces a transient cAMP signal and that this response requires a functional RAS1 or RAS2 gene (Mbonyi et al. 1988; Thevelein 1988). The cAMP signal activates $\mathrm{CAPK}$; for $A D H 2$, glucose repression occurs via this pathway, as CAPK appears to phosphorylate and inactivate the $A D H 2$ transcriptional activator ADR1 (Cherry et al. 1989; Taylor and Young 1990). Cherry et al. (1989) have also shown that mutations in BCY1 (encoding the regulatory subunit of $\mathrm{CAPK}$ ) reduce expression of $A D H 2$. The result that a mutation in $S$. pombe cgs1 (encoding the regulatory subunit of cAPK) reduces $f b p 1$ derepression indicates that the role of cAMP in glucose repression of $f b p 1$ is to stimulate cAPK. (Interestingly, the ability of a git2-1 cgs1-1 strain to show mild derepression of $f b p 1-l a c Z$ expression (Table 3 ) suggests that glucose also causes repression of $f b p 1$ transcription by a cAMP-independent mechanism.)

From our results thus far, we cannot conclude that cAPK acts to repress $f b p 1$ transcription by the same mechanism as seen for $A D H 2$ in S. cerevisiae. Mutations in git6 are not suppressed by exogenous cAMP or by a mutation in cgs 1 (Fig. 3; Table 3). Possibly, git6 encodes the catalytic subunit of CAPK that inactivates the transcriptional activator of $f b p 1$. However, the git 6 gene could also encode a transcriptional repressor that is activated by cAPK. The cAMP-responsive element-binding protein (CREB) from rat can act as either a transcriptional activator or a repressor, depending on its phosphorylation state, at the c-iun promoter (Lamph et al. 1990).

Previous work has shown that transcription of the mei2 gene of $S$. pombe is also repressed by cAMP (Watanabe et al. 1988). This gene is required for entry into meiosis and is inducible by nitrogen starvation; however, the role of cAMP in meiosis is still unclear (for review, see McLeod 1989). Therefore, S. pombe may regulate cAMP levels with respect to both the carbon source and the presence of a nitrogen source. It is likely that additional factors are involved that distinguish between response to glucose and response to a nitrogen source.

Our future work will address how adenylate cyclase is activated in $S$. pombe and how cAPK acts to repress $f b p 1$ transcription. By continued molecular and genetic analyses of git genes, and by analysis of the $f b p 1$ promoter, we hope to further our understanding of the role of signal transduction and transcriptional repression of $f b p 1 \mathrm{ex}-$ pression.

\section{Materials and methods}

Yeast strains and media

All S. pombe strains used in this study are listed in Table 4. Genetic nomenclature of $S$. pombe follows rules proposed by Kohli (1987). The fbp $1:$ :ura4 allele is a translational fusion that disrupts the $f b p 1$ gene, and the ura $4:: f b p 1-l a c Z$ allele is a disruption of the ura 4 gene by the $f b p 1-l a c Z$ translational fusion (Hoffman and Winston 1990). Standard rich media YEA and YEL (Gutz et al. 1974) were supplemented with $2 \%$ casamino acids. Minimal media (SD) supplemented with amino acids and synthetic complete media (SC) lacking a specific amino acid (Sherman et al. 1978) were used with modifications described previously (Hoffman and Winston 1990). PM medium (defined minimal medium; Watanabe et al. 1988) was supplemented with required amino acids. Carbon sources were generally present at a concentration of $3 \%$, and strains were grown at $30^{\circ} \mathrm{C}$, unless otherwise specified. Sensitivity to 5FOA (PCR, Inc.) was determined on SC, SC-leu, and SC-ade solid media containing $8 \%$ glucose, $50 \mathrm{mg} / \mathrm{liter}$ of uracil, and $0.4 \mathrm{~g} / \mathrm{liter}$ of $5 \mathrm{FOA}$. When indicated, cAMP (Aldrich Chemical Company, Inc.) was added to a final concentration of $5 \mathrm{~mm}$. Crosses were done on either MEA (Gutz et al. 1974) containing $0.4 \%$ glucose or on YPD (Sherman et al. 1978).

\section{Recombinant DNA methodology}

Standard recombinant DNA techniques (including DNA restriction digests, ligations, and bacterial transformations) were done according to Maniatis et al. (1982). E. coli strains HB101 (Boyer and Roulland-Dussoix 1969) and JM101 (Messing 1979) were the host strains for bacterial transformations. Yeast transformations were done by the lithium acetate method (Ito et al. 1983). Small-scale plasmid preparations from $E$. coli were done by the alkaline lysis method (Birnboim and Doly 1979). Smallscale DNA preparations from yeast to recover plasmids in $E$. coli were done as described previously (Hoffman and Winston 1987). DNA fragment isolation was done by electroelution. Restriction endonucleases SalI and XhoI were purchased from Boe- 
Table 4. Strains

\begin{tabular}{|c|c|}
\hline Strain & Genotype \\
\hline CHP1 & $h^{+}$ade6-M216 lys1-131 leu1-32 ura4::fbp1-lacZ fbp1::ura4 git1-1 \\
\hline FWP148 & $h^{+}$lys1-131 leu1-32 ura4::fbp1-lacZ fbp1::ura4 [pCHY26] \\
\hline FWP167 & $h^{+}$ade6-M210 his7-366 leu1-32 ura4::fbp1-lacZ fbp1::ura4 [pCHY27] \\
\hline FWP101 & $h^{+}$ade6-M210 his7-366 leu1-32 ura4::fbp1-lacZ fbp1::ura4 \\
\hline FWP112 & $h^{-}$ade6-M216 his7-366 leu1-32 ura4::fbp1-lacZ fbp1::ura4 \\
\hline FWP113 & $h^{+}$ade6-M210 his7-366 leu1-32 ura4::fbp1-lacZ fbp1::ura4 git1-1 \\
\hline CHP210 & $h^{-}$ade6-M216 his7-366 leu1-32 ura4::fbp1-lacZ fbp1::ura4 git2-210 \\
\hline FWP189 & $h^{+}$ade6-M210 his7-366 leu1-32 ura4::fbp1-lacZ fbp1::ura4 git2 ${ }^{+}$int::LEU2 \\
\hline FWP190 & $h^{+}$ade6-M210 his7-366 leu1-32 ura4::fbp1-lacZ fbp1::ura4 git2-1 int::LEU2 \\
\hline CHP200 & $h^{-}$ade6-M216 his7-366 leu1-32 ura4::fbp1-lacZ fbp1::ura4 git3-200 \\
\hline CHP17 & $h^{+}$ade6-M210 his7-366 leu1-32 ura4::fbp1-lacZ fbp1::ura4 git4-17 \\
\hline CHP75 & $h^{+}$ade6-M210 his7-366 leu1-32 ura4::fbp1-lacZ fbp1::ura4 git5-75 \\
\hline CHP261 & $h^{-}$ade6-M216 his7-366 leu1-32 ura4::fbp1-lacZ fbp1::ura4 git6-261 \\
\hline CHP235 & $h^{-}$ade6-M216 his7-366 leu1-32 ura4::fbp1-lacZ fbp1::ura4 git7-235 \\
\hline CHP60 & $h^{+}$ade6-M210 his7-366 leu1-32 ura4::fbp1-lacZ fbp1::ura4 git8-60 \\
\hline $\mathrm{CHP} 232$ & $h^{-}$ade6-M216 his7-366 leu1-32 ura4::fbp1-lacZ fbp1::ura4 git9-232 \\
\hline CHP201 & $h^{-}$ade6-M216 his7-366 leu1-32 ura4::fbp1-lacZ fbp1::ura4 git10-201 \\
\hline FWP70 & $h^{+}$ade6-M216 lys1-131 leu1-32 ura4::fbp1-lacZ \\
\hline FWP134 & $h^{+}$ade6-M210 leu1-32 ura4::fbp1-lacZ git1-1 \\
\hline FWP135 & $h^{-}$his7-366 leu1-32 ura4::fbp1-lacZ git2-210 \\
\hline FWP136 & $h^{-}$ade6-M216 his7-366 leu1-32 ura4::fbp1-lacZ git3-200 \\
\hline FWP173 & $h^{-}$leu1-32 ura4::fbp1-lacZ git4-17 \\
\hline FWP174 & $h^{-}$leu1-32 ura4::fbp1-lacZ git5-75 \\
\hline FWP139 & $h^{-}$ade6-M216 his7-366 leu1-32 ura4::fbp1-lacZ git6-261 \\
\hline FWP140 & $h^{-}$leu1-32 ura4::fbp1-lacZ git7-235 \\
\hline FWP175 & $h^{-}$leu1-32 ura4::fbp1-lacZ git8-60 \\
\hline FWP142 & $h^{+}$leu1-32 ura4::fbp1-lacZ git9-232 \\
\hline FWP143 & $h^{+}$leu1-32 ura4::fbp1-lacZ git10-201 \\
\hline FWP182 & $h^{-}$leu1-32 ura4::fbp1-lacZ git2-7 \\
\hline FWP183 & $h^{-}$leu1-32 ura4::fbp1-lacZ git2-13 \\
\hline FWP184 & $h^{-}$leu1-32 ura4::fbp1-lacZ git2-61 \\
\hline FWP191 & $h^{-}$ade6-M210 leu1-32 ura4::fbp1-lacZ git2-1 int::LEU2 \\
\hline CHP7 & $h^{+}$ade6-M210 his7-366 leu1-32 ura4::fbp1-lacZ fbp1::ura4 git2-7 \\
\hline $\mathrm{CHP} 13$ & $h^{+}$ade6-M210 his7-366 leu1-32 ura4::fbp1-lacZ fbp1::ura4 git2-13 \\
\hline CHP61 & $h^{+}$ade6-M210 his7-366 leu1-32 ura4::fbp1-lacZ fbp1::ura4 git2-61 \\
\hline FWP114 & $h^{-}$ade6-M216 his7-366 leu1-32 ura4::fbp1-lacZ fbp1::ura4 git2-7 \\
\hline FWP169 & $h^{-}$ade6-M216 his7-366 leu1-32 ura4::fbp1-lacZ fbp1::ura4 git2-61 \\
\hline FWP177 & $h^{90}$ ade6-M210 leu1-32 cgs1-1 \\
\hline FWP180 & $h^{+}$ade6-M216 leu1-32 ras $1^{+}$int: $: L E U 2 B g I I I$ \\
\hline FWP179 & $h^{+}$ade6-M216 leu1-32 ura4- ras1- int::LEU2HincII \\
\hline FWP181 & $h^{90}$ ade6-M210 leu1-32 ras1 ${ }^{\text {val17 }}$ int : LEU2Bg1II \\
\hline FWP192 & $h^{90}$ ade6-M216 leu1-32 ura4::fbp1-lacZ cgs1-1 \\
\hline FWP193 & $h^{+}$ade6-M216 leu1-32 his7-366 ura4::fbp1-lacZ cgs1-1 git2-1 int::LEU2 \\
\hline FWP194 & $h^{+}$ade6-M216 leu1-32 his7-366 ura4::fbp1-lacZ cgs1-1 git6-261 \\
\hline
\end{tabular}

hringer Mannheim Biochemicals. T4 DNA polymerase, the large fragment of DNA polymerase I (Klenow), DNA primers, and all other restriction enzymes were purchased from New England Biolabs.

\section{Cloning of the git2 gene}

The git2 gene was cloned as a high copy number suppressor of the git1-1 mutation in strain CHPl (Table 4). CHPl was transformed to $\mathrm{Leu}^{+}$on SC-leu (8\% glucose) with an $S$. pombe genomic library (partial HindIII-digested DNA cloned into the HindIII site of pWH5; Wright et al. 1986; P. Young and D. Beach, unpubl.), and transformants were replica-plated to SC-leu and SC-leu with 5FOA to screen for plasmids that restored regulation of the $f b p 1-u r a 4$ fusion. Plasmids were isolated from 10
$5 \mathrm{FOA}^{\mathrm{R}}$ transformants and used to transform E. coli strain $\mathrm{HB} 101$ to $\mathrm{Amp}^{\mathrm{R}}$. Plasmid preparations from the $E$. coli transformants were used for restriction analyses and retransformation of CHP1. A single candidate plasmid, pCHY26 (Fig. 1), conferred a strong $5 \mathrm{FOA}^{\mathrm{R}}$ phenotype on retransformation of $\mathrm{CHP1}$. Other candidate plasmids conferred a weak $5 \mathrm{FOA}^{\mathrm{R}}$ phenotype and have not been examined further.

Plasmid pCHY26 was integrated into the $S$. pombe genome by transformation of CHP1 with PmII-digested pCHY26. Analysis of transformants indicated that pCHY26 did not carry the git1 gene, as originally anticipated. In a cross, the $\mathrm{Git}^{-}$phenotype associated with the git1-1 mutation and the Leu ${ }^{+}$phenotype associated with the LEU2 gene from pCHY26 segregated independently in tetrads. Additional genetic crosses with a progeny from this cross, FWP148 $\left(\mathrm{Git}^{+} \mathrm{Leu}^{+}\right)$, however, demonstrated 
that these phenotypes did cosegregate (nine of nine PD tetrads) when crossed with the git2 mutant CHP210. Although this genetic analysis indicated that pCHY26 carried the git2 gene, we were unable to determine whether pCHY26 had integrated by homologous recombination (as judged by Southern hybridization analysis) owing to the large size of the insert DNA in this plasmid. To determine whether the plasmid insert directs homologous integration at git2, we constructed plasmid pCHY27 (Fig. 1) by digesting pCHY26 with $\mathrm{BamHI}$ and recircularizing the large restriction fragment. Plasmid pCHY27 contains most of the vector DNA plus $\sim 4 \mathrm{~kb}$ of $S$. pombe insert DNA. Homologous integration into strain FWP101 $\left(\mathrm{Git}^{+} \mathrm{Leu}^{-}\right)$, creating strain FWP167 ( $\mathrm{Git}^{+} \mathrm{Leu}^{+}$), was confirmed by Southern hybridization analysis (Southern 1975) of both EcoRI and EcoRV digests of transformant DNA. In a cross of FWP167 with strain CHP210, the $\mathrm{Git}^{+}$and $\mathrm{Leu}^{+}$phenotypes cosegregated (25 PD tetrads and one TT tetrad). Therefore, pCHY27 contains genomic DNA from the git2 locus.

\section{Construction of the git2-1 null allele}

A git2 null allele was constructed by digesting pCHY26 with $B g I I I$ and recircularizing the large BgIII fragment, resulting in plasmid pCHY28 (Fig. 1). This plasmid is unable to complement mutations in git2. On the basis of DNA sequence analysis of the git2 gene (cyr1; Yamawaki-Kataoka et al. 1989; Young et al. 1990 ) this BglII deletion removes part of git2 that encodes the adenylate cyclase catalytic domain and shifts the remainder of the coding region out of frame with the amino-terminal coding region. Plasmid pCHY28 was digested with ClaI, followed by ligation of the large restriction fragment, resulting in removal of much of the vector DNA. This plasmid, pCHY29, contains a single Sall site, present in the insert DNA. pCHY29 was then digested with SalI and ligated with a 2.2-kb SalI-Xhol fragment from YEp13 (Broach et al. 1979), which carries the LEU2 gene of $S$. cerevisiae, to form plasmid pCHY30. (The LEU2 gene of $S$. cerevisiae complements the leu1-32 mutation in $S$. pombe.) Strain FWP101 was transformed to $\mathrm{Leu}^{+}$with a HindIII fragment from pCHY30 that carries the git2 BgIII deletion (git2-1) and the LEU2 insertion. One transformant (FWP190) was confirmed to contain a single copy of the git2-1 null allele and the adjacent $L E U 2$ insertion by Southern hybridization analysis. In a cross between FWP190 $\left(\mathrm{Git}^{-} \mathrm{Leu}^{+}\right)$and FWP112 (Git $\left.{ }^{+} \mathrm{Leu}^{-}\right)$, the Git ${ }^{-}$phenotype cosegregates with the Leu ${ }^{+}$phenotype. The git2-1 mutation is also unable to complement other git2 mutations (Fig. 4). Strain FWP189, which contains the LEU2 insertion, but adjacent to git2 ${ }^{+}$, is $\mathrm{Git}^{+}$. In addition, integration of the LEU2 gene adjacent to the git2 ${ }^{+}$gene has no effect on expression of an $f b p 1-l a c Z$ fusion (Table 3; strain FWP189); therefore, it is the git2-1 deletion and not the LEU2 insertion that causes the $\mathrm{Git}^{-}$phenotype.

\section{Complementation analysis}

Complementation analyses were carried out by determining the sensitivity or resistance to 5FOA of diploids formed between git2 mutant strains, as described previously (Hoffman and Winston 1990). Complementation between two recessive git2 alleles restores regulated expression of the $f b p 1-u r a 4$ fusion, resulting in a $5 F O A^{R}$ phenotype when grown under repressing conditions $(8 \%$ glucose). Noncomplementation results in constitutive expression of the fbp1-ura4 fusion, resulting in a $5 \mathrm{FOA}^{\mathrm{s}}$ phenotype. Diploids formed between any two members of the two groups that displayed git2 intragenic complementation showed partial to complete $5 \mathrm{FOA}^{\mathrm{R}}$ (complementation), whereas diploids formed between members of the same groups were $5 \mathrm{FOA}^{\mathrm{s}}$ (noncomplementation).

\section{B-Galactosidase assays and Northern hybridization analysis}

$\beta$-Galactosidase assays and Northern hybridization analysis were done as described previously (Hoffman and Winston 1990). Plasmid pAV06 (Vassarotti and Friesen 1985) was used as a probe for the $f b p 1$ transcript. RNA amounts were standardized by hybridization to plasmid pYK311, which carries the leu1 gene (Kikuchi et al. 1988).

\section{cAMP assays}

Extracts for cAMP assays were prepared from cultures grown to $1 \times 10^{7}$ cells $/ \mathrm{ml}$ in PM medium according to the method of Fedor-Chaiken et al. (1990) and assayed using the radioimmune assay kit available from Amersham. cAMP levels were standardized to total protein concentration using the BCA protein assay (Smith et al. 1985). Total protein extracts were made in $0.2 \mathrm{~N} \mathrm{NaOH}$ by vortexing cells in the presence of glass beads.

\section{Adenylate cyclase assays}

Adenylate cyclase activity was assayed in membrane extracts as described by Casperson et al. (1985) with some modifications. Strains were grown in $250 \mathrm{ml}$ of YEL ( $8 \%$ glucose) to a density of $\sim 1 \times 10^{7}$ cells $/ \mathrm{ml}$. The cultures were poured over an equal volume of ice and subsequently maintained at $0^{\circ} \mathrm{C}$ to optimize recovery of active adenylate cyclase. Cells were pelleted and washed twice, once with $30 \mathrm{ml}$ water and once with $10 \mathrm{ml}$ of buffer A [50 mM Mes (pH 6.2), 1 mM $\mathrm{MgCl}_{2}, 1 \mathrm{~mm}$ EGTA, $1 \mathrm{~mm}$ $\beta$-mercaptoethanol], and resuspended in $0.4 \mathrm{ml}$ of buffer $A$ with $1 \mathrm{mM}$ PMSF. Glass beads were added to the meniscus, and cells were lysed by vortex-mixing for $4 \mathrm{~min}$ (done in 30-sec intervals followed by at least $1 \mathrm{~min}$ on ice). Lysates were transferred to Eppendorf tubes (glass beads were washed with $1 \mathrm{ml}$ of buffer A and pooled with the lysates) and centrifuged for $15 \mathrm{~min}$ in a microfuge. Membrane extracts were gently collected by resuspending the transluscent section of the pellet in $100 \mu \mathrm{l}$ of buffer A with $10 \%$ glycerol and were stored at $-70^{\circ} \mathrm{C}$.

Each reaction mix contained $20-25 \mu \mathrm{g}$ of membrane extracts in $100 \mathrm{~mm}$ Mes (pH 6.2), $5 \mathrm{~mm} \mathrm{MnCl}, 0.1 \mathrm{mg} / \mathrm{ml}$ of BSA, $20 \mathrm{mM}$ creatine phosphate, 20 units $/ \mathrm{ml}$ of creatine phosphokinase, 0.1 mM EGTA, 2 mM $\beta$-mercaptoethanol, $1.3 \mathrm{mM}\left[\alpha^{32}\right.$ P]ATP $\{20-65$ $\mathrm{cpm} / \mathrm{pmole}$ ), and $0.5 \mathrm{mM}{ }^{3} \mathrm{H}$-labeled cAMP (5000 cpm). Each reaction was initiated by the addition of the membrane extract, proceeded for $30 \mathrm{~min}$ at $30^{\circ} \mathrm{C}$, and was terminated by the addition of $1 \mathrm{ml}$ of $0.2 \%$ SDS, $0.1 \mathrm{mM}$ cAMP, and $1.2 \mathrm{mM} \mathrm{ATP,}$ followed by boiling for $3 \mathrm{~min}$. The amount of ${ }^{32} \mathrm{p}$-labeled cAMP produced was determined by the procedure of Salomon et al. (1974). Assays were linear with respect to time and membrane extract concentration under these conditions.

\section{Acknowledgments}

We thank Maureen McLeod for sharing unpublished data and for providing strains carrying the cgs 1-1 and ras1 mutations, Masayuki Yamamoto for sharing unpublished data, and David Beach for the $S$. pombe genomic library used to clone the git2 gene. We thank Scott Cameron, Eva Neer, Ken Ferguson, Yuriko Yamawaki-Kataoka, and Hye-Ryun Choe for helpful discussions regarding adenylate cyclase activity and in vitro assays during the course of these studies. We also thank Greg Prelich, Karen Arndt, and Elizabeth Malone for critical reading of this manuscript, and David Eisenmann and Greg Prelich for assis- 
tance in preparation of the figures. This work was supported by American Cancer Society postdoctoral grant PF-2853 to C.S.H., and National Science Foundation grant DCB8451649 and a grant from the Stroh Brewery Company to F.W.

The publication costs of this article were defrayed in part by payment of page charges. This article must therefore be hereby marked "advertisement" in accordance with 18 USC section 1734 solely to indicate this fact.

\section{References}

Beebe, S.J. and J.D. Corbin. 1986. Cyclic nucleotide protein kinases. In The enzymes (ed. P.D. Boyer), vol. 17, pp. 43-111. Academic Press, New York.

Birnboim, H.C. and J. Doly. 1979. A rapid alkaline extraction procedure for screening recombinant plasmid DNA. Nucleic Acids Res. 7: 1513-1523.

Boeke, J.D., F. Lacroute, and G.R. Fink. 1984. A positive selection for mutants lacking orotidine-5'-phosphate decarboxylase activity in yeast: 5-Fluoro-orotic acid resistance. Mol. Gen. Genet. 197: 345-346.

Botsford, J.L. 1981. Cyclic nucleotides in procaryotes. Microbiol. Rev. 45: 620-642.

Boyer, H.W. and D. Roulland-Dussoix. 1969. A complementation analysis of the restriction and modification of DNA in E. coli. J. Mol. Biol. 41: 458-472.

Broach, J.R., J.N. Strathern, and J.B. Hicks. 1979. Transformation in yeast: Development of a hybrid cloning vector and isolation of the CAN1 gene. Gene 8: 121-133.

Broek, D., N. Samiy, O. Fasano, A. Fujiyama, F. Tamanoi, J. Northup, and M. Wigler. 1985. Differential activation of yeast adenylate cyclase by wild-type and mutant ras proteins. Cell 41: 763-769.

Broek, D., T. Toda, T. Micheali, L. Levin, C. Birchmeier, M. Zoller, S. Powers, and M. Wigler. 1987. The S. cerevisiae $C D C 25$ gene product regulates the RAS/adenylate cyclase pathway. Cell 48: 789-799.

Casperson, G.F., N. Walker, and H.R. Bourne. 1985. Isolation of the gene encoding adenylate cyclase in Saccharomyces cerevisiae. Proc. Natl. Acad. Sci. 82: 5060-5063.

Cherry, J.R., T.R. Johnson, C. Dollard, J.R. Shuster, and C.L. Denis. 1989. Cyclic AMP-dependent protein kinase phosphorylates and inactivates the yeast transcriptional activator ADR1. Cell 56: 409-419.

Edelman, A.M., D.K. Blumenthal, and E.G. Krebs. 1987. Protein serine/threonine kinases. Annu. Rev. Biochem. 56: 567-613.

Engelberg, D., E. Poradosu, G. Simchem, and A. Levitzki. 1990. Adenylyl cyclase activity of the fission yeast Schizosaccharomyces pombe is not regulated by guanyl nucleotides. FEBS Lett. 261: 413-418.

Eraso, P. and J.M. Gancedo. 1984. Catabolite repression in yeast is not associated with low levels of cAMP. Eur. J. Biochem. 141: 195-198.

Fedor-Chaiken, M., R.J. Deschenes, and J.R. Broach. 1990. $S R V 2$, a gene required for $R A S$ activation of adenylate cyclase in yeast. Cell 61: 329-340.

Field, J., A. Vojtek, R. Ballester, G. Bolger, J. Colicelli, K. Ferguson, J. Gerst, T. Kataoka, T. Michaeli, S. Powers, M. Riggs, L. Rodgers, I. Wieland, B. Wheland, and M. Wigler. 1990a. Cloning and characterization of $C A P$, the $S$. cerevisiae gene encoding the $70 \mathrm{kd}$ adenylyl cyclase-associated protein. Cell 61: $319-327$.

Field, J., H.-P. Xu, T. Michaeli, R. Ballester, P. Sass, M. Wigler, and J. Colicelli. 1990b. Mutations of the adenylyl cyclase gene that block RAS function in Saccharomyces cerevisiae.
Science 247: 464-467.

Fukui, Y. and Y. Kaziro. 1985. Molecular cloning and sequence analysis of a ras gene from Schizosaccharomyces pombe. EMBO I. 4: 687-691.

Fukui, Y., T. Kozasa, Y. Kaziro, T. Takeda, and M. Yamamoto. 1986. Role of a ras homolog in the life cycle of Schizosaccharomyces pombe. Cell 44: 329-336.

Gancedo, J.M. and C. Gancedo. 1986. Catabolite repression mutants of yeast. FEMS Microbiol. Rev. 32: 179-187.

Gilman, A.G. 1984. G proteins and dual control of adenylate cyclase. Cell 36: 577-579.

Gutz, H., H. Heslot, U. Leupold, and N. Loprieno. 1974. Schizosaccharomyces pombe. In Handbook of Genetics led. R.C. King), pp. 395-446. Plenum Press, New York.

Hoffman, C.S. and F. Winston. 1987. A ten-minute DNA preparation from yeast efficiently releases autonomous plasmids for transformation of Escherichia coli. Gene 57: 267-272.

-1989. A transcriptionally regulated expression vector for the fission yeast Schizosaccharomyces pombe. Gene 84: 473-479.

-1990. Isolation and characterization of mutants constitutive for expression of the $f b p 1$ gene of Schizosaccharomyces pombe. Genetics 124: 807-816.

Ito, H., Y. Fukuda, K. Murata, and A. Kimura. 1983. Transformation of intact yeast cells treated with alkali cations. $J$. Bacteriol. 153: 163-168.

Johnston, M. 1987. A model fungal gene regulatory mechanism: The GAL genes of Saccharomyces cerevisiae. Microbiol. Rev. 51: 458-476.

Kawamukai, M., K. Ferguson, M. Wigler, and D. Young. 1991. Genetic and biochemical analysis of the adenylyl cyclase of Schizosaccharomyces pombe. Cell regul. (in press).

Kikuchi, Y., Y. Kitazawa, H. Shimatake, and M. Yamamoto. 1988. The primary structure of the leu $1^{+}$gene of Schizosaccharomyces pombe. Curr. Genet. 14: 375-379.

Kohli, J. 1987. Genetic nomenclature and gene list of the fission yeast Schizosaccharomyces pombe. Curr. Genet. 11: 575589.

Lamph, W.W., V.J. Dwarki, R. Ofir, M. Montminy, and I.M. Verma. 1990. Negative and positive regulation by transcription factor cAMP response element-binding protein is modulated by phosphorylation. Proc. Natl. Acad. Sci. 87: 43204324.

Levitzki, A. 1988. From epinephrine to cyclic AMP. Science 241: 800-806.

Maeda, T., N. Mochizuki, and M. Yamamoto. 1990. Adenylyl cyclase is dispensible for vegetative cell growth in fission yeast Schizosaccharomyces pombe. Proc. Natl. Acad. Sci. 87: 7814-7818.

Magasanik, B. 1961. Catabolite repression. Cold Spring Harbor Symp. Quant. Biol. 26: 249-256.

Maniatis, T., E.F. Fritsch, and J. Sambrook. 1982. Molecular cloning: A laboratory manual. Cold Spring Harbor Laboratory, Cold Spring Harbor, New York.

Matsumoto, K., I. Uno, Y. Oshima, and T. Ishikawa. 1982a. Isolation and characterization of yeast mutants deficient in adenylate cyclase and cAMP-dependent protein kinase. Proc. NatI. Acad. Sci. 79: 2355-2359.

Matsumoto, K., I. Uno, A. Toh-e, T. Ishikawa, and Y. Oshima. 1982b. Cyclic AMP may not be involved in catabolite repression in Saccharomyces cerevisiae: Evidence from mutants capable of utilizing it as an adenine source. I. Bacteriol. 150: $277-285$.

Matsumoto, K., I. Uno, T. Ishikawa, and Y. Oshima. 1983. Cyclic AMP may not be involved in catabolite repression in Saccharomyces cerevisiae: Evidence from mutants unable 
to synthesize it. J. Bacteriol. 156: 898-900.

Mbonyi, K., M. Beullens, K. Detremerie, L. Geerts, and J.M. Thevelein. 1988. Requirement of one functional RAS gene and inability of an oncogenic ras variant to mediate the glucose-induced cyclic AMP signal in the yeast Saccharomyces cerevisiae. Mol. Cell. Biol. 8: 3051-3057.

McLeod, M. 1989. Regulation of meiosis: From DNA binding protein to protein kinase. Bioessays 11: 9-14.

Messing, J. 1979. A multipurpose cloning system based on the single-stranded bacteriophage M13. Recomb. DNA Tech. Bull. 1: 43-44.

Nadin-Davis, S.A., R.C.A. Yang, S.A. Narang, and A. Nasim. 1986a. The cloning and characterization of a $R A S$ gene from Schizosaccharomyces pombe. J. Mol. Evol. 23: 41-51.

Nadin-Davis, S.A., A. Nasim, and D. Beach. 1986b. Involvement of ras in sexual differentiation but not in growth control in fission yeast. EMBO /. 5: 2963-2971.

Nadin-Davis, S.A., A. Nasim, Y. Fukui, and M. Yamamoto. 1989. Oncogene homologs. In Molecular biology of the fission yeast (ed. A. Nasim, P. Young, and B.F. Johnson), pp. 97-126. Academic Press, New York.

Nikawa, J., S. Cameron, T. Toda, K.M. Ferguson, and M. Wigler. 1987. Rigorous feedback control of cAMP levels in Saccharomyces cerevisiae. Genes \& Dev. 1: 931-937.

Robinson, L., J. Gibbs, M. Marshall, I. Sigal, and K. Tatchell. 1987. $C D C 25$ : A component of the $R A S$-adenylate cyclase pathway in Saccharomyces cerevisiae. Science 235: 12181221.

Roesler, W.J., G.R. Vandenbark, and R.W. Hanson. 1988. Cyclic AMP and the induction of eukaryotic gene transcription. $J$. Biol. Chem. 263: 9063-9066.

Saloman, Y., C. Londos, and M. Rodbell. 1974. A highly sensitive adenylate cyclase assay. Anal. Biochem. 58: 541-548.

Smith, P.K., R.I. Krohn, G.T. Hermanson, A.K. Mallia, F.H. Gartner, M.D. Provenzano, E.K. Fujimoto, N.M. Goeke, B.J. Olsen, and D.C. Klenk. 1985. Measurement of protein using bicinchoninic acid. Anal. Biochem. 150: 76-85.

Sherman, F., G.R. Fink, and C.W. Lawrence. 1978. Cold Spring Harbor Laboratory manual. Methods in yeast genetics, Cold Spring Harbor Laboratory, Cold Spring Harbor, New York.

Southern, E.M. 1975. Detection of specific sequences among DNA fragments separated by gel electrophoresis. I. Mol. Biol. 98: 503-517.

Tanaka, K., K. Matsumoto, and A. Toh-e. 1989. IRA1: An inhibitory regulator of the RAS/cyclic AMP pathway in Saccharomyces cerevisiae. Mol. Cell. Biol. 9: 757-768.

Tanaka, K., M. Nakafuku, F. Tamanoi, Y. Kaziro, K. Matsumoto, and A. Toh-e. 1990. IRA2, a second gene of Saccharomyces cerevisiae that encodes a protein with a domain homologous to mammalian ras GTPase-activating protein. Mol. Cell. Biol. 10: 4303-4313.

Taylor, W.E. and E.T. Young. 1990. cAMP-dependent phosphorylation and inactivation of yeast transcription factor ADR1 does not affect DNA binding. Proc. Natl. Acad. Sci. 87: 4098-4102.

Thevelein, J.M. 1988. Regulation of trehalase activity by phosphorylation-dephosphorylation during developmental transitions in fungi. Exp. Mycol. 12: 1-12.

Toda, T., I. Uno, T. Ishikawa, S. Powers, T. Kataoka, D. Broek, S. Cameron, J. Broach, K. Matsumoto, and M. Wigler. 1985. In yeast, RAS proteins are controlling elements of adenylate cyclase. Cell 40: 27-36.

Toda, T., S. Cameron, P. Sass, M. Zoller, J.D. Scott, B. McMullen, M. Hurwitz, E.G. Krebs, and M. Wigler. 1987a. Cloning and characterization of $B C Y 1$, a locus encoding a regulatory subunit of the cAMP dependent protein kinase in
Saccharomyces cerevisiae. Mol. Cell. Biol. 7: 1371-1377.

Toda, T., S. Cameron, P. Sass, M. Zoller, and M. Wigler. 1987b. Three different genes in $S$. cerevisiae encode the catalytic subunits of the cAMP-dependent protein kinase. Cell 50: $277-287$.

Vassarotti, A. and J.D. Friesen. 1985. Isolation of the fructose1,6-bisphosphatase gene of the yeast Schizosaccharomyces pombe. J. Biol. Chem. 260: 6348-6353.

Watanabe, Y., Y. Iino, K. Furuhata, C. Shimoda, and M. Yamamoto. 1988. The $S$. pombe mei2 gene encoding a crucial molecule for commitment to meiosis is under the regulation of cAMP. EMBO J. 7: 761-767.

Wright, A., K. Maundrell, W.D. Heyer, D. Beach, and P. Nurse. 1986. Vectors for the construction of gene banks and the integration of cloned genes in Schizosaccharomyces pombe and Saccharomyces cerevisiae. Plasmid 15: 156-158.

Yamawaki-Kataoka, Y., T. Tamaoki, H.-R., Choe, H. Tanaka, and T. Kataoka. 1989. Adenylate cyclases in yeast: A comparison of the genes from Schizosaccharomyces pombe and Saccharomyces cerevisiae. Proc. Natl. Acad. Sci. 86: 56935697.

Young, D., M. Riggs, J. Fields, A. Vojtek, D. Broek, and M. Wigler. 1990. The adenylyl cyclase gene from Schizosaccharomyces pombe. Proc. Natl. Acad. Sci. 86: 7989-7993. 


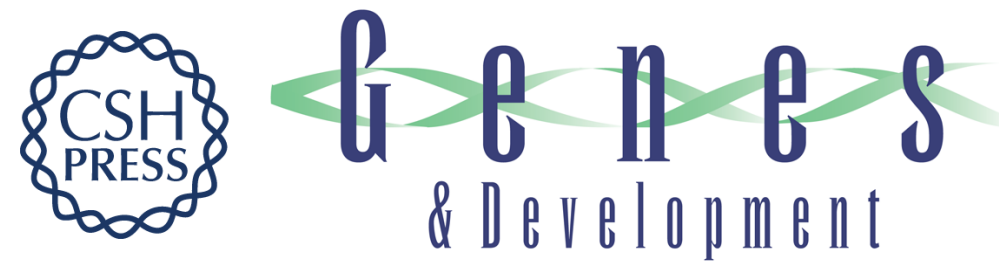

\section{Glucose repression of transcription of the Schizosaccharomyces pombe fbp1 gene occurs by a cAMP signaling pathway.}

C S Hoffman and F Winston

\section{Genes Dev. 1991, 5:}

Access the most recent version at doi:10.1101/gad.5.4.561

$\begin{array}{ll}\text { References } & \begin{array}{l}\text { This article cites } 56 \text { articles, } 23 \text { of which can be accessed free at: } \\ \text { http://genesdev.cshlp.org/content/5/4/561.full.html\#ref-list-1 }\end{array}\end{array}$

License

Email Alerting

Service

Receive free email alerts when new articles cite this article - sign up in the box at the top right corner of the article or click here.

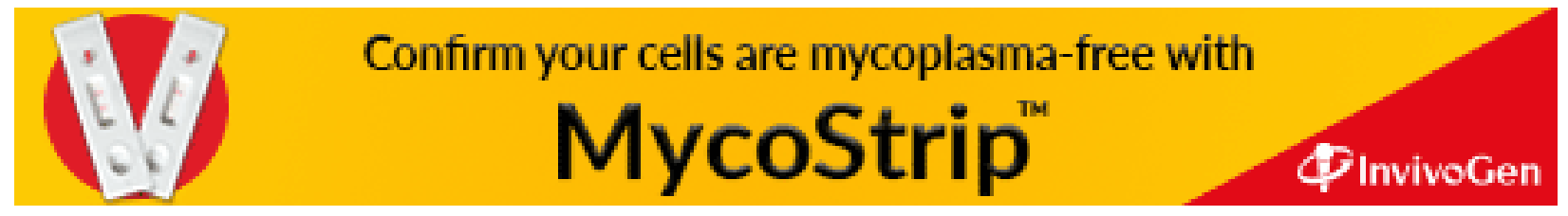

